\title{
Prevalencia de Sintomatología de Salud Mental y Hábitos de Salud en una Muestra de Universitarios Chilenos
}

\section{Prevalence of Mental Health Symptoms and Health-Related Habits in a Sample of Chilean University Students}

\author{
Ana Barrera-Herrera ${ }^{1}$ y Yusting San Martín² \\ ${ }^{1}$ Departamento de Psicología, Universidad Católica de Temuco \\ ${ }^{2}$ Servicio de Salud Estudiantil, Dirección de Desarrollo Estudiantil, Universidad de La Frontera
}

\begin{abstract}
El presente estudio tuvo por objetivo describir la prevalencia de sintomatología de salud mental y hábitos de salud, y analizar diferencias según sexo, procedencia y nivel socioeconómico (NSE) en una muestra por conveniencia de 601 estudiantes de 3 universidades chilenas de Arica, Concepción y Temuco. Los participantes respondieron la $1^{\text {a }}$ Encuesta de Salud Mental Universitaria, la cual estuvo compuesta por 6 instrumentos de salud mental que miden variables sociodemográficas y cuadros de salud mental más frecuentes en esta población. A través de análisis de frecuencias y diferencias de grupos ( $t$ de Student y ANOVA), los resultados revelan que más de un $45 \%$ de los participantes presenta sintomatología de riesgo asociada a depresión, ansiedad, o estrés, existiendo comorbilidad. Las mujeres presentan mayor ansiedad y estrés, mientras que los jóvenes de procedencia urbana, mayor ansiedad. Un 5\% presenta ideación suicida y un 14\% revela conductas alimentarias de riesgo, siendo estas más prevalentes en mujeres. Además, un 50\% reporta síntomas de insomnio y un $42 \%$, hipersomnia diurna, siendo esta última más frecuente en jóvenes urbanos. Existe un variado y preocupante consumo de sustancias, siendo los varones los que presentan mayor consumo en tabaco, alcohol, marihuana y alucinógenos, mientras que las mujeres consumen más tranquilizantes, constatando también diferencias en tabaco y alcohol según procedencia y NSE. Estas cifras corroboran la alta prevalencia de diferentes problemas de salud mental entre los universitarios de esta muestra, señalando la necesidad de profundizar su estudio e implementar programas de promoción, prevención y tratamiento oportuno en este grupo.
\end{abstract}

Palabras clave: salud mental, prevalencia, estudiantes universitarios, adultez emergente, Chile

The present study aimed to describe the prevalence of mental health symptoms and health-related habits and analyze differences by sex, origin, and socioeconomic status (SES) in a convenience sample of 601 students from 3 Chilean universities in the cities of Arica, Concepción, and Temuco. Participants answered the $1^{\text {st }}$ University Mental Health Survey, which was composed of 6 mental health instruments that measure sociodemographic variables and the mental health conditions most frequently observed in this population. Analyses of frequencies and group differences (Student's $t$-test and ANOVA) indicate that more than $45 \%$ of the participants display risk symptoms associated with depression, anxiety, or stress, with comorbidity. Women exhibit greater anxiety and stress, while young people from urban backgrounds show greater anxiety. Five percent display suicidal ideation and $14 \%$ report risky eating behaviors, with the latter being more prevalent in women. In addition, $50 \%$ of participants report symptoms of insomnia and $42 \%$, daytime hypersomnia, with the latter being more frequent in urban youth. There is a varied and worrying level of substance consumption, with men reporting the highest consumption of tobacco, alcohol, marijuana, and hallucinogens, while women consume more tranquilizers. Differences in tobacco and alcohol use were also found in connection with background and SES. These findings corroborate the high prevalence of several mental health problems among the university students in this sample, demonstrating the need to conduct further research on these issues and implement promotion, prevention, and early treatment programs for this population group.

Keywords: mental health, prevalence, university students, emerging adulthood

\section{Ana Barrera-Herrera (iD https://orcid.org/0000-0003-0574-6568}

Yusting San Martín (iD https://orcid.org/0000-0001-5700-5126

Esta investigación está inserta en el Proyecto CONICYT PAI 77170108, titulado "Estudio nacional de prevalencia de trastornos de salud mental y hábitos de salud, y su relación con dimensiones de la adultez emergente, en jóvenes universitarios chilenos". La investigación fue financiada por la Comisión Nacional de Investigación Científica y Tecnológica y por la Iniciativa Científica Milenio del Ministerio de Economía, Fomento y Turismo, proyecto NCS17_035, titulado "Núcleo Milenio para Mejorar la Salud Mental de Adolescentes y Jóvenes, Imhay". Las autoras agradecen la participación de los universitarios que conformaron la muestra de la investigación y que permitieron realizar el estudio, además de reconocer el trabajo de tesistas de pregrado de psicología involucrados en el proceso de investigación.

La correspondencia relativa a este artículo debe ser dirigida a Ana Barrera-Herrera, Departamento de Psicología, Universidad Católica de Temuco, Manuel Montt 056, Temuco, Chile. Email: abarrera@uct.cl 
Según la Organización Mundial de la Salud ([OMS], 2013), en el mundo existen más de 400 millones de personas que sufren de un trastorno mental o de la conducta. Estos problemas de salud mental afectan a la sociedad en su totalidad, no obstante, existen ciertos grupos que presentan más riesgo de experimentar este tipo de trastornos, a saber, las personas vulnerables económicamente, migrantes, mujeres, adultos mayores y las personas con baja escolaridad (Departamento de Salud Mental y Abuso de Sustancias, 2004). Respecto a este último punto, la evidencia empírica de los últimos 10 años ha llevado a cuestionar el factor protector de los años de escolaridad en salud mental, puesto que estudios de prevalencia con población universitaria, tanto en Chile como a nivel internacional, revelan que los jóvenes que cursan estudios universitarios han presentado en los últimos años un aumento sostenido en la prevalencia de trastornos mentales (Auerbach et al., 2016; Storrie et al., 2010). Asimismo, se ha observado que este grupo presenta comorbilidades en salud mental y que, en muchos casos, la prevalencia de trastornos como depresión, ansiedad y riesgo suicida supera a las cifras obtenidas tanto para la población general como para jóvenes no universitarios (Arrieta Vergara et al., 2014; Baader et al., 2014; Departamento de Epidemiología, 2018; Micin \& Bagladi, 2011; Stallman \& Shochet, 2009).

Este preocupante y complejo escenario puede explicarse por varios factores. En primer lugar, los universitarios transitan por un nuevo periodo evolutivo, el cual ha sido acuñado por el investigador Jeffrey Arnett (2000, 2007/2008) como adultez emergente. Esta etapa se sitúa entre los 18 y 29 años y se distingue de otros periodos por cinco características particulares que también están presentes en universitarios chilenos: la exploración de la identidad, la inestabilidad, una etapa de gran optimismo y posibilidades, centrarse en sí mismo y sentirse entre la adolescencia y la adultez (Arnett, 2000, 2004). Todas estas características conforman una etapa de vida compleja que comprende una serie de retos para los jóvenes en torno a la adaptación a actividades, como la vida laboral o universitaria, el desafío de independizarse progresivamente de los padres, formar nuevos vínculos significativos y preparar el sinuoso camino a la adultez (Arnett, 2007/2008; Barrera-Herrera \& Vinet, 2017).

Además de las características distintivas de este periodo vital, un segundo factor relevante que explicaría este fenómeno corresponde a las características del entorno universitario. Durante su trayectoria académica, los jóvenes se enfrentan a un sistema académico exigente, muy distinto al sistema de educación secundaria (Álamo et al., 2017; Micin \& Bagladi, 2011). Es en este nuevo contexto donde deben asumir nuevas responsabilidades académicas, organizar adecuadamente su tiempo para responder a las múltiples tareas, fomentar su autonomía y tener las competencias necesarias para enfrentarse a estresores, tales como abandonar el núcleo familiar, la inestabilidad económica, responder a expectativas personales y familiares, compatibilizar estudios con crianza y/o trabajo, entre otros (Baader et al., 2014; Barrera-Herrera \& Vinet, 2017; Micin \& Bagladi, 2011).

Frente a este escenario, la necesidad de adaptarse a una nueva etapa de vida y las demandas propias del contexto universitario, son factores que hacen de la adultez emergente un periodo especialmente inestable, que puede propiciar la aparición de sintomatología o trastornos de salud mental (Arnett et al., 2014; Baader et al., 2014; Micin \& Bagladi, 2011; Schulenberg et al., 2004).

En Chile, en los últimos 10 años ha surgido un importante marco de investigaciones realizadas con universitarios en el área de salud mental, las cuales se han centrado en estudiar la prevalencia de los trastornos más comunes, tales como depresión, ansiedad, estrés, trastornos adaptativos, trastornos de la conducta alimentaria, consumo de sustancias y riesgo suicida (Antúnez \& Vinet, 2013; Arrieta Vergara et al., 2014; Baader et al., 2014; Barraza et al., 2015; Cova Solar et al., 2007; Durán-Agüero et al., 2016; Florenzano, 2005; Jerez-Mendoza \& Oyarzo-Barría; 2015; Micin \& Bagladi, 2011, Pérez-Villalobos et al., 2012; Servicio Nacional para la Prevención y Rehabilitación del Consumo de Drogas y Alcohol [SENDA], 2019).

Especial énfasis ha recibido el estudio de la sintomatología de depresión, ansiedad y estrés, por su alta incidencia en población universitaria (Antúnez \& Vinet, 2013; Arrieta Vergara et al., 2014; Baader et al., 2014; Micin \& Bagladi, 2011). Al respecto, la evidencia disponible pone de manifiesto que entre el 16,5 y el 30,1\% de los universitarios chilenos presenta sintomatología depresiva (Antúnez \& Vinet, 2013; Baader et al., 2014; Pérez-Villalobos et al., 2012; Rossi et al., 2019). En cuanto a ansiedad y estrés, las cifras siguen tendencias similares, probablemente por la similitud entre estos cuadros: para ansiedad, las cifras oscilan entre un 20,9 y un 38,8\% (Antúnez \& Vinet, 2013; Pérez-Villalobos et al., 2012), mientras que para estrés se reporta una prevalencia de $32 \%$, considerando niveles medios a moderados de estrés percibido (Barraza et al., 2015). Además de estas preocupantes cifras, el interés por estos tres cuadros sintomatológicos se hace más relevante porque la evidencia señala que dichos cuadros tienden a presentarse en comorbilidad y, a su vez, aumentan el riesgo de 
desarrollar otros problemas de salud mental (Román et al., 2016), como trastornos en la conducta alimentaria, consumo perjudicial de alcohol y riesgo de suicidio (Baader et al., 2014).

No obstante la existencia de estos importantes estudios en salud mental universitaria, al analizar la información recabada por estas investigaciones, surgen algunas dificultades: (a) a pesar de estudiar los mismos problemas de salud mental, la prevalencia reportada en cada investigación suele ser distinta, puesto que algunos estudios han analizado sintomatología de salud mental mientras que otros reportan prevalencia de trastorno clínico, lo que implica el uso de instrumentos distintos, tales como escalas de screening o entrevistas diagnósticas, respectivamente; (b) los estudios mencionados realizan diferenciaciones de sintomatologías según variables sociodemográficas como sexo o nivel socioeconómico (NSE) de los sujetos; sin embargo, podrían existir otras variables de relevancia para la salud mental en el periodo de adultez emergente que no han sido consideradas, tal como la procedencia de los estudiantes; (c) considerando la diversidad de patologías de salud mental existentes en esta población, se hace necesario desarrollar estudios que puedan evaluar distintas problemáticas y que obtengan una fotografía actualizada del estado de salud mental de los estudiantes y (d) ciertas problemáticas de salud mental, tales como problemas de sueño y conductas de riesgo alimentarias, han recibido menos atención por parte de las investigaciones analizadas y, por tanto, es necesario incluirlas en las investigaciones.

En razón de lo expuesto, el presente estudio, inserto en el Proyecto CONICYT PAI77170108, tuvo por objetivo describir la prevalencia de sintomatología de salud mental y hábitos de salud en una muestra de universitarios chilenos y detectar posibles diferencias según variables sociodemográficas de relevancia en este periodo vital. Además, dada la alta comorbilidad entre las sintomatologías de depresión, ansiedad y estrés, como objetivo secundario se evaluó el efecto mediador de la sintomatología de depresión en la asociación entre los cuadros de ansiedad y estrés.

\section{Método}

\section{Diseño}

Esta investigación fue de carácter cuantitativo, un estudio descriptivo de tipo transversal, con un alcance descriptivo-correlacional, puesto que se describe en un primer lugar la prevalencia obtenida en cada instrumento en un momento específico y luego se analizan posibles diferencias según variables sociodemográficas de relevancia para la etapa de adultez emergente.

\section{Participantes}

A través de un muestreo no probabilístico por conveniencia, la muestra estuvo integrada por 601 estudiantes chilenos de diferentes carreras de pregrado de tres universidades pertenecientes al Consejo de Rectores, de las ciudades de Arica (Universidad de Tarapacá; 41,3\%), Concepción (Universidad de Concepción; 24,6\%) y Temuco (Universidad Católica de Temuco; 34,1\%). Los estudiantes pertenecían a carreras de diferentes facultades, existiendo una mayor representación de carreras vinculadas al área de ciencias sociales $(56,1 \%)$, educación $(16,1 \%)$, ingeniería $(12,3 \%)$ y, en un porcentaje menor, carreras relacionadas a las áreas de agronomía (6\%), salud (4,2\%), arte (3\%) y derecho (2,3\%). Los criterios de inclusión fueron: encontrarse entre el rango de edad de 18 a 29 años y tener nacionalidad chilena.

El rango de edad de los participantes fue de 18 a 29 años, con un promedio de edad de 20,7 años ( $D E=2,29$ ). Un $63,4 \%$ de la muestra eran mujeres y un $36,6 \%$, hombres. Un $88,5 \%$ de los estudiantes provenía de zonas urbanas aledañas a las universidades de pertenencia, mientras que un 11,5\% provenía de zonas rurales. En relación al NSE, calculado a partir del nivel educacional y ocupación del jefe o jefa de hogar (Adimark, 2000), un 29,7\% pertenecía al NSE medio, seguido por un 25\% de NSE medio-bajo, un 23,5\% de NSE medio-alto, un $10,5 \%$ de NSE alto, un 5,8\% de NSE bajo y, finalmente, un 5,5\% de NSE muy bajo. Además, la mayor parte de los estudiantes vivía con sus padres (59,7\%) y no tenían hijos (93,5\%). Un 21,8\% de los jóvenes complementaba sus estudios superiores con algún trabajo remunerado de 16 horas a la semana en promedio $(D E=8)$.

$\mathrm{Al}$ indagar sobre la salud mental de los estudiantes, los universitarios reportaron que un $44 \%$ ha estado en tratamiento psicológico alguna vez en su vida, mientras que un $56 \%$ no ha requerido o asistido a este tipo de tratamientos. Al momento de la aplicación del instrumento, un 11,3\% reportó estar en tratamiento psicológico. 
BARRERA-HERRERA Y SAN MARTÍN

Los participantes fueron contactados a través de informantes clave, como profesores o directores pertenecientes a diversas carreras de las universidades mencionadas, quienes autorizaron realizar la aplicación de la encuesta durante el periodo de clases.

\section{Instrumentos}

Para esta investigación se construyó la $1^{\text {a }}$ Encuesta de Salud Mental Universitaria, la cual estuvo compuesta por un set de instrumentos de salud mental, destacando los siguientes:

\section{Cuestionario Sociodemográfico}

Es un cuestionario de autorreporte construido por el equipo de investigación, el cual contiene preguntas que permitieron caracterizar la muestra, como edad, sexo, procedencia, NSE, entre otras.

\section{Escalas de Depresión, Ansiedad y Estrés (DASS-21; Antúnez \& Vinet, 2012)}

Este instrumento de autorreporte, validado y estandarizado para universitarios chilenos, tiene como propósito evaluar la frecuencia e intensidad en que los síntomas de depresión, ansiedad y estrés se experimentan durante la última semana. Consta de 21 ítems con cuatro alternativas de respuesta en formato Likert, las cuales van desde 0 (No describe nada de lo que me pasó o sentí en la semana) hasta 3 (Sí, esto me pasó mucho o casi siempre). Cada escala contiene siete ítems. Un ejemplo para la Escala de Depresión es el ítem "No podía sentir nada positivo", para la escala de Ansiedad, "Sentí miedo sin saber por qué" y para la escala de Estrés, "Me costó mucho calmarme". El puntaje por subescala puede variar entre 0 y 21 puntos, por lo que a mayor puntaje obtenido se estima que existe mayor presencia de sintomatología depresiva, ansiosa y de estrés. Asimismo, el instrumento posee puntos de corte establecidos por Román et al. (2016), que permiten discriminar a la población con sintomatología clínica y que requiere de apoyo profesional. Así, este estudio estableció que para las escalas de Depresión y Estrés el puntaje de corte es mayor a 5 y para la escala de Ansiedad es mayor a 4.

Este instrumento posee óptimos niveles de confiabilidad en población universitaria chilena (Antúnez \& Vinet, 2012; Mellor et al., 2015; Román et al., 2016). Además, se ha establecido su validez de constructo mediante análisis factorial exploratorio, el que arrojó una estructura de tres factores, explicando el 49,99\% de la varianza total (Antúnez \& Vinet, 2012). También este instrumento posee evidencia de su validez concurrente y divergente, utilizando para ello el Inventario de Depresión de Beck-II y el Inventario de Ansiedad de Beck (Antúnez \& Vinet, 2012; Román Mella et al., 2014).

Respecto de la consistencia interna, en este estudio el instrumento para la escala total obtuvo un alfa de Cronbach de 0,93, mientras que para sus dimensiones de Depresión, Ansiedad y Estrés, uno de 0,88, 0,79 y 0,86 , respectivamente.

Inventario de Ideación Suicida Positiva y Negativa (PANSI; Villalobos-Galvis, 2010, Basado en la Versión Original de Osman et al., 2003)

Este instrumento de autorreporte fue diseñado para evaluar la ideación suicida (IS). Está compuesto por un total de 14 ítems, los cuales se dividen en las escalas de Ideación Suicida Positiva (6 ítems; e.g., "¿Tuviste esperanza en el futuro porque las cosas estaban saliendo como tu querías?") e Ideación Suicida Negativa (8 ítems; e.g., "¿Pensaste que tus problemas eran tan graves que la única opción que tenías era suicidarte?"), cuyos ítems son puntuados según su frecuencia de aparición en un intervalo de dos semanas. La escala de IS positiva aborda pensamientos protectores contra el suicidio, relacionados a la capacidad de control, esperanza por el futuro y autoconfianza, mientras que la escala de IS negativa está integrada por ítems de riesgo suicida, como desesperanza, soledad y evaluación del suicidio como opción. Cada ítem se evalúa en una escala tipo Likert de cinco puntos que oscila entre 0 (nunca) y 4 (siempre). Además, basado en estudios previos, se han definido puntajes de corte que indican la necesidad de una evaluación adicional del riesgo suicida. Para la escala de IS negativa este puntaje es $>2$ y para la escala de IS positiva es < 3,33 (Osman et al., 2003).

Respecto a las propiedades psicométricas de este instrumento, cabe señalar que a pesar de que no está validado en Chile, ha obtenido adecuadas propiedades psicométricas en estudios con muestras similares a universitarios. En una investigación con estudiantes colombianos de enseñanza secundaria y superior, se obtuvo una óptima consistencia interna tanto para la escala total $(\alpha=0,90)$, como para las subescalas de IS 
negativa y positiva ( $\alpha=0,93$ y 0,84 respectivamente). En este mismo estudio, se otorga evidencia sobre la validez de constructo del instrumento, al demostrar relaciones directas con medidas de depresión, desesperanza e ideas suicidas, y relaciones inversas con una escala de autoestima (Villalobos-Galvis, 2010).

En la presente investigación, para la escala total se obtuvo un índice de consistencia interna, evaluada con alfa de Cronbach, de 0,66, en tanto que para las subescalas de IS positiva y negativa se obtuvieron alfas de 0,82 y 0,95 , respectivamente.

\section{Cuestionario Breve de Conductas Alimentarias de Riesgo (CBCAR; Unikel-Santoncini et al., 2004)}

Este instrumento tiene por objetivo medir las conductas características de los trastornos alimentarios (TCA), tomando como referencia los criterios diagnósticos que explicita el DSM IV. Se integra por 10 preguntas que indagan la frecuencia de conductas específicas, tales como atracones y purgas, medidas compensatorias, como pastillas y laxantes, y medidas restrictivas, como dietas y ejercicio, considerando un rango de tiempo de 3 meses previos a la aplicación del cuestionario. Posee cuatro opciones de respuesta en una escala tipo Likert que va desde Nunca o casi nunca (0), Algunas veces (1), Frecuentemente (2) y Muy frecuentemente (3). A mayor puntaje, mayor riesgo de TCA. Los autores han propuesto una clasificación de tres niveles de riesgo, según los siguientes puntajes: (a) Sin riesgo: 0 a 6 puntos, (b) Riesgo moderado: 7 a 10 puntos y (c) Riesgo alto: sobre 10 puntos (Altamirano-Martinez et al., 2011).

Actualmente este instrumento no cuenta con validación en Chile, sin embargo, ha presentado adecuadas características psicométricas en una muestra de mujeres mexicanas estudiantes y de pacientes diagnosticadas de TCA, obteniendo una consistencia interna de $a=0,83$ para la escala total, mientras que para las tres escalas que lo componen, la consistencia interna fue de 0,74 (Atracón-Purga), 0,72 (Medidas Compensatorias) y 0,76 (Restricción) (Unikel-Santoncini et al., 2004). En el mismo estudio, se utilizaron instrumentos acordes a lo expresado en la literatura respecto a la comorbilidad con que se presentan los TCA con los trastornos afectivos y la mortalidad asociada al suicidio, realizando análisis de correlación con las escalas de sintomatología depresiva e ideación suicida, corroborando la validez concurrente del cuestionario. Adicionalmente, se estableció la validez predictiva de este instrumento mediante un análisis discriminante (Unikel-Santoncini et al., 2004).

En el presente estudio, el instrumento obtuvo un alfa de Cronbach de 0,72 , mientras que la escala de Atracón-Purga uno de 0,59, la de Medidas Compensatorias uno de 0,45 y la de Restricción uno de 0,70.

\section{Cuestionario Oviedo del Sueño (COS; Bobes García et al., 2000)}

Consiste en una entrevista semiestructurada de ayuda diagnóstica que tiene por propósito detectar trastornos como insomnio e hipersomnia, según los criterios del DSM IV y CIE 10, considerando síntomas presentes en el último mes. Consta de 15 ítems que se agrupan en tres escalas: Satisfacción Subjetiva del Sueño (1 ítem), Insomnio (9 ítems) e Hipersomnia (3 ítems). La mayor parte de los ítems contienen cinco opciones de respuesta en formato Likert, los cuales solicitan información referente a frecuencia, calidad y tiempo de sueño. La puntuación de la subescala de Satisfacción Subjetiva del Sueño oscila entre 1-7, la de Insomnio entre 9-45 y la de Hipersomnia va de 1-15 puntos. Para estimar la prevalencia de las escalas de Insomnio e Hipersomnia, se ha utilizado la media de 22,9 y 7,4 , respectivamente.

Este instrumento no se encuentra validado en Chile, sin embargo, estudios desarrollados en España han analizado sus propiedades psicométricas y han demostrado su adecuada confiabilidad, evidencias de validez convergente y adecuada capacidad de discriminación (Bobes García et al., 2000; García-Portilla et al., 2009). A modo de ejemplo, en el estudio de García-Portilla et al. (2009), desarrollado con personas mayores a 18 años con un trastorno mental grave y otro grupo control sin enfermedades de salud mental, se obtuvo un alfa de Cronbach de 0,90 para el instrumento total, mientras que para la escala de Insomnio fue de 0,91 y para la escala de Hipersomnia, 0,88. Asimismo, en esta investigación se estableció la validez convergente de la escala, a partir de niveles aceptables de correlación entre las puntuaciones del instrumento COS y otras medidas del ritmo sueño-vigilia que evaluaban trastornos del sueño, alteraciones del sueño, extrema reducción del sueño y no dormir. Además, se determinó la validez discriminante del cuestionario, al evaluar diferencias entre pacientes y controles, entre distintos niveles de gravedad del trastorno mental y entre diferentes niveles de gravedad del trastorno del sueño. 
En esta investigación este instrumento presentó adecuados índices de consistencia interna: para la escala total y para la subescala de Insomnio se obtuvo un alfa de Cronbach de 0,72 y para la escala de Hipersomnia se obtuvo un alfa de 0,80 .

\section{Prueba de Detección de Consumo de Alcohol, Tabaco y Sustancias (ASSIST; Área de Desarrollo Sostenible y Salud Ambiental, 2011)}

Este instrumento proporciona información sobre el consumo de sustancias a lo largo de la vida, así como el consumo y los problemas relacionados a este en los últimos 3 meses. Consta de ocho preguntas y se sugiere ser aplicado por un profesional de la salud. Debido a la complejidad de aplicación y extensión del instrumento, en este estudio solo se aplicó un extracto de la escala, que evalúa la frecuencia de consumo de diversas sustancias en los últimos 3 meses, en una escala Likert de cinco opciones, que van desde Nunca (0) hasta Diariamente o casi diariamente (4). Según refiere la OMS (Área de Desarrollo Sostenible y Salud Ambiental, 2011), el ASSIST ha sido validado en población adulta (entre 18 y 60 años de edad) y ha demostrado ser culturalmente neutral (Soto-Brandt et al., 2014). Para esta investigación se utilizó el extracto de la versión 3.0 validada en Chile para mayores de 18 años, la que en su estudio de validación demostró buenas propiedades psicométricas, obteniendo una alta consistencia interna con un alfa de Cronbach para la escala total de 0,91 (Soto-Brandt et al., 2014). Asimismo, el estudio entrega evidencia de validez concurrente, que se respalda en una alta correlación entre el ASSIST y otros instrumentos que evalúan el consumo de sustancias (ASI-Lite, SDS, MINI-Plus, AUDIT y RTQ-Smoking).

En el presente estudio, este extracto del instrumento ASSIST ha obtenido una confiabilidad de 0,63.

\section{Procedimiento}

Antes de realizar la aplicación, se explicó a los alumnos de forma breve el propósito de la investigación, destacando el carácter voluntario y confidencial de su participación. La invitación tuvo una buena acogida por parte de la mayoría de los estudiantes, lo que propició que cerca de un $99 \%$ de los alumnos invitados accedieran a participar de la investigación, motivados por la temática y su relevancia en el periodo universitario; solo se excluyó un par de casos debido a motivaciones personales o al no cumplimiento de los criterios de inclusión. Luego, se les entregó el consentimiento informado, documento visado por el Comité de Ética de la Universidad Católica de Temuco, y, después de firmarlo, se aplicó individualmente el instrumento en un período promedio de 40 minutos aproximadamente, durante el año 2018.

Cabe señalar que, como la $1^{\text {a }}$ Encuesta de Salud Mental Universitaria recoge datos sensibles asociados a la salud mental, como resguardo ético en el consentimiento informado se explicitó a los estudiantes que quienes estuvieran interesados en ser informados sobre algún puntaje de riesgo en las diferentes escalas contenidas en la encuesta, podían anotar voluntariamente su correo electrónico al final de esta para ser notificados y recibir la recomendación de consultar con un especialista en salud mental. Así, una vez concluida la recolección de datos por universidad, se detectaron los cuestionarios de riesgo y aquellos universitarios que habían proporcionado su correo electrónico fueron notificados oportunamente (en su mayoría, los cuestionarios de riesgo contenían un correo de contacto).

\section{Análisis de Datos}

Primeramente, se ingresaron los datos en el software estadístico SPSS v. 25, donde se realizaron análisis preliminares para evaluar la calidad de los datos (por ejemplo, búsqueda de datos perdidos, casos fuera de rango o datos inconsistentes a través de análisis de frecuencias). Luego, se calcularon los puntajes de las escalas utilizadas y se realizaron análisis descriptivos para caracterizar la muestra, tales como promedios y porcentajes. Asimismo, se estimó la consistencia interna de los instrumentos utilizados por medio de alfa de Cronbach.

Posteriormente, se utilizaron pruebas paramétricas para comparar la sintomatología de los distintos instrumentos utilizados, según sexo, NSE y procedencia, utilizando en la mayor parte de los análisis solo los casos con sintomatología de riesgo o clasificando a los sujetos según el nivel de riesgo, para analizar tales diferencias en profundidad. Para estimar la diferencia según sexo y procedencia se utilizó $t$ de Student y para comparar según NSE (seis grupos, desde NSE bajo a NSE alto) se realizó un análisis de varianza (ANOVA) para grupos independientes. A fin de complementar tales análisis, se estimó el tamaño del efecto por medio de los estadísticos $d$ de Cohen y Eta al cuadrado ( $\left.\mathfrak{y}^{2}\right)$. 
Adicionalmente, dada la alta comorbilidad y correlación entre los componentes del instrumento DASS-21 y para responder al objetivo secundario del estudio, se realizó un análisis de senderos para probar el efecto mediador de la sintomatología de depresión en la asociación entre los cuadros de ansiedad y estrés. Para ello, se estimó efecto directo, indirecto y total y además se calculó el $r^{2}$ del modelo total. Este análisis fue realizado con el software STATA v. 14.2 .

\section{Resultados}

\section{Depresión, Ansiedad y Estrés}

A partir del instrumento DASS-21, se pudo constatar que un 46\% de la muestra presentó síntomas depresivos, un 45,5\%, síntomas ansiosos y un 53,5\%, sintomatología de estrés. Si se considera comorbilidad, es decir, estudiantes que poseen puntajes sobre el punto de corte en las tres escalas, un 29,7\% presenta los tres tipos de sintomatología.

Al realizar análisis de diferencias de grupo en las tres sintomatologías según la variable sexo, los resultados revelan que las mujeres presentan puntajes de riesgo más elevados en Ansiedad y Estrés. Por su parte, en la escala de Depresión no hubo diferencias según sexo (ver Tabla 1).

Luego se analizaron posibles diferencias según la procedencia de los sujetos, esto es, si provienen de zonas urbanas o rurales, encontrando que solo para la escala de Ansiedad existen diferencias, revelando que los universitarios de zonas urbanas presentaron medias más altas en esta dimensión (ver Tabla 1).

En cuanto al NSE, la prueba ANOVA mostró que no hubo diferencias para las escalas de Depresión, $F(5,250)=0,367, p=0,871$, Ansiedad, $F(5,255)=1,463, p=0,203$, y Estrés, $F(5,293)=1,524, p=0,182$.

\section{Ideación Suicida}

De acuerdo a los resultados obtenidos, en relación a la escala de IS positiva, un $24,3 \%$ de la muestra ha presentado factores protectores ante la IS, relacionados con la sensación de control sobre su vida y esperanza sobre el presente y futuro. No existen diferencias en esta dimensión, al comparar los resultados según sexo, $t(143)=0,703, p=0,483$, procedencia, $t(144)=1,082, p=0,281$, y NSE de los sujetos, $F(5,129)=1,197$, $p=0,314$. Respecto de la dimensión de IS negativa, un $5,1 \%$ de los estudiantes ha presentado en las últimas dos semanas pensamientos o IS, lo que corresponde a 31 estudiantes, no existiendo diferencias según sexo, $t(29)=-0,187, p=0,853$, y NSE, $F(5,23)=0,873, p=0,514$. Respecto a procedencia, no fue posible realizar la comparación, pues los casos con IS negativa solo provenían de la zona urbana.

\section{Conductas Alimentarias de Riesgo}

Los resultados señalan que, de acuerdo a las categorías de riesgo, la mayor parte de los estudiantes no presenta conductas alimentarias de riesgo, mientras que existe un porcentaje pequeño con riesgo alto. Este último grupo manifiesta la presencia de variadas conductas de riesgo que podrían constituir un trastorno de la conducta alimentaria (ver Tabla 2).

\section{Tabla 1}

Prueba t de Student para Diferencias de Sintomatología, según Sexo y Procedencia

\begin{tabular}{lrrrrrrr}
\hline \multicolumn{1}{c}{ DASS-21 } & $M(D E)$ & $M(D E)$ & \multicolumn{1}{c}{$t$} & $p$ & g.l. & 95\% IC & $d$ de Cohen \\
\hline \multicolumn{1}{c}{ Sexo } & \multicolumn{1}{c}{ Hombre } & \multicolumn{1}{c}{ Mujer } & & & & & \\
\hline Depresión & $10,64(4,13)$ & $10,37(3,98)$ & 0,53 & 0,596 & 273 & & \\
Ansiedad & $8,01(3,42)$ & $9,05(3,61)$ & $-2,16$ & 0,032 & 283 & {$[-1,96,-0,09]$} & $-0,29$ \\
Estrés & $0,48(3,32)$ & $10,51(3,65)$ & $-2,36$ & 0,015 & 318 & {$[-1,88,-0,17]$} & $-0,29$ \\
\hline Procedencia & Urbano & Rural & & & & & \\
\hline Depresión & $10,56(4,09)$ & $9,40(2,98)$ & 1,37 & 0,171 & 273 & & \\
Ansiedad & $8,93(3,58)$ & $7,48(3,44)$ & 2,13 & 0,034 & 282 & {$[0,10,2,78]$} & 0,41 \\
Estrés & $10,35(3,52)$ & $9,31(3,92)$ & 1,71 & 0,087 & 319 & & \\
\hline
\end{tabular}


BARRERA-HERRERA Y SAN MARTÍN

Tabla 2

Porcentaje de Niveles de Riesgo de Conductas Alimentarias para la Muestra Total y según Sexo

\begin{tabular}{lccr}
\hline \multirow{2}{*}{ CBCAR } & Muestra total & Hombres & \multicolumn{1}{c}{ Mujeres } \\
\cline { 2 - 4 } & $n(\%)$ & $n(\%)$ & $n(\%)$ \\
\hline Sin riesgo & $331(50,4)$ & $144(43,5)$ & $187(56,5)$ \\
Riesgo moderado & $184(33,8)$ & $56(30,4)$ & $128(69,6)$ \\
Riesgo alto & $83(15,7)$ & $19(22,9)$ & $64(77,1)$ \\
\hline
\end{tabular}

Nota. Sin información: 3 casos (1 hombre y 2 mujeres).

Los análisis de diferencias de grupo muestran que no existen diferencias en cuanto al sexo y procedencia de los universitarios para estos tres niveles de riesgo (ver Tabla 3). Sin embargo, al considerar la muestra total, el análisis revela que las mujeres tienen mayores conductas alimentarias de riesgo. Esto no ocurre para la variable procedencia.

Por otro lado, no se observan diferencias según NSE para ninguno de los tres grupos, a saber: sin riesgo, $F(5,302)=0,953, p=0,447$, riesgo moderado, $F(5,164)=1,064, p=0,382$, y riesgo alto, $F(5,69)=1,140$, $p=0,348$. Asimismo, tampoco se encontraron diferencias al considerar la muestra total, $F(5,547)=1,197, p=0,309$.

\section{Calidad del Sueño}

Los estudiantes reportaron dormir en promedio 6,4 horas $(D E=1,36)$, presentando un rango de horas de sueño de 3 a 12 horas, lo que demuestra una gran variabilidad en esta dimensión. Un $36,8 \%$ se encuentra satisfecho/a con su calidad del sueño, un 33\% se considera ni satisfecho/a ni insatisfecho/a, mientras que un $30,2 \%$ se encuentra insatisfecho/a. Un antecedente relevante es que un 49,9\% reporta tener síntomas asociados a insomnio, esto es, dificultades para conciliar el sueño o tener un sueño reparador. Además, un $42 \%$ de los estudiantes presentó síntomas relacionados a la hipersomnia, esto es, excesivo sueño durante la mayor parte del día.

Respecto a los análisis de comparación de grupos, en la escala Hipersomnia hubo diferencias solo según la procedencia de los sujetos, siendo los universitarios de procedencia urbana los que tuvieron puntuaciones más altas (ver Tabla 4). Respecto al NSE, no hubo diferencias, $F(5,229)=0,899, p=0,482$. Por su parte, en la escala Insomnio no se reportaron diferencias según sexo, procedencia (ver Tabla 4) y NSE, $F(5,269)=0,556, p=0,733$.

Tabla 3

Prueba t de Student para Diferencias de Niveles de Riesgo CBCAR, según Sexo y Procedencia

\begin{tabular}{lrrrrrrr}
\hline \multicolumn{1}{c}{ CBCAR } & \multicolumn{1}{c}{$M(D E)$} & \multicolumn{1}{c}{$M(D E)$} & \multicolumn{1}{c}{$t$} & \multicolumn{1}{c}{ g.l. } & 95\% IC & d de Cohen \\
\hline \multicolumn{1}{c}{ Sexo } & \multicolumn{1}{c}{ Hombre } & \multicolumn{1}{c}{ Mujer } & & & & & \\
\hline Sin riesgo & $3,30(1,86)$ & $3,57(1,80)$ & $-1,35$ & 0,177 & 329 & & \\
Riesgo moderado & $8,23(1,25)$ & $8,49(1,13)$ & $-1,39$ & 0,166 & 182 & & \\
Riesgo alto & $13,42(4,06)$ & $13,94(2,99)$ & $-0,66$ & 0,546 & 81 & & \\
Muestra total & \multicolumn{1}{c}{$5,44(3,82)$} & $6,98(4,26)$ & $-4,56$ & $<0,001$ & 496 & {$[-2,21,-0,88]$} & 0,38 \\
\hline \multicolumn{1}{c}{ Procedencia } & \multicolumn{1}{c}{ Urbano } & \multicolumn{1}{c}{ Rural } & & & & & \\
\hline Sin riesgo & $3,51(1,85)$ & $3,07(1,61)$ & 1,47 & 0,141 & 330 & & \\
Riesgo moderado & $8,42(1,16)$ & $8,26(1,24)$ & 0,54 & 0,588 & 180 & \\
Riesgo alto & $13,81(3,30)$ & $13,57(2,76)$ & 0,18 & 0,857 & 82 & \\
Muestra total & $6,52(4,20)$ & $5,57(3,90)$ & 1,78 & 0,070 & 596 & & \\
\hline
\end{tabular}


Tabla 4

Prueba t de Student para Escalas de Sueño, según Sexo y Procedencia

\begin{tabular}{lccccccc}
\hline Escalas de sueño & $M(D E)$ & $M(D E)$ & $t$ & $p$ & g.l. & 95\% IC & $d$ de Cohen \\
\hline \multicolumn{1}{c}{ Sexo } & Hombre & Mujer & & & & & \\
\hline Insomnio & $27,66(4,34)$ & $28,94(4,06)$ & $-0,74$ & 0,460 & 296 & & \\
Hipersomnia & $10,21(1,78)$ & $10,56(1,96)$ & $-1,33$ & 0,183 & 249 & & \\
\hline \multicolumn{1}{c}{ Procedencia } & Urbano & Rural & & & & & \\
\hline Insomnio & $27,84(4,05)$ & $28,37(4,80)$ & $-0,67$ & 0,505 & 297 & & \\
Hipersomnia & $10,57(1,95)$ & $9,76(1,55)$ & 2,55 & 0,015 & 40 & {$[0,16,1,44]$} & 0,46 \\
\hline
\end{tabular}

\section{Consumo de Sustancias}

En términos de frecuencia de consumo, las drogas que se reportaron con mayor consumo semanal fueron el alcohol, luego la marihuana y en tercer lugar el tabaco. Un dato que llama la atención es que un 9\% de los estudiantes reporta un consumo ocasional de tranquilizantes, ya sea prescritos o autoadministrados. Además, un $3,2 \%$ reporta haber consumido ocasionalmente cocaína (ver Tabla 5).

En cuanto al análisis de diferencias en el consumo de estas drogas, la prueba $t$ de Student revela que existen diferencias por sexo en el consumo de tabaco, alcohol, marihuana, alucinógenos y tranquilizantes, existiendo en las primeras cuatro drogas un mayor consumo por parte de los universitarios varones, mientras que las mujeres tienen un mayor consumo de tranquilizantes (ver Tabla 6). En relación a la procedencia de los sujetos, las pruebas $t$ de Student indican que existen diferencias en el consumo de tabaco y alcohol, siendo los universitarios de zonas urbanas los que presentan mayor consumo.

Respecto al NSE, la prueba ANOVA revela que solo existen diferencias en el alcohol, $F(5,544)=3,075$, $p=0,010, \mathrm{n}^{2}=0,02$. La prueba post hoc de Tukey señala que las diferencias se encuentran entre los jóvenes que pertenecen al NSE medio bajo y muy alto, $t(544)=-2,922, p=0,042$, siendo los universitarios de NSE muy alto los que reportan un mayor consumo. Respecto a las sustancias restantes, no se reportan diferencias por NSE en tabaco, $F(5,547)=0,114, p=0,989$, marihuana, $F(5,545)=0,686, p=0,634$, cocaína, $F(5,546)=0,678$, $p=0,640$, tranquilizantes, $F(5,546)=1,854, p=0,101$, y alucinógenos, $F(5,545)=0,773, p=0,570$.

Tabla 5

Prevalencia de Consumo de Drogas, según Tipo

\begin{tabular}{lccccc}
\hline & Nunca & Ocasional & Mensual & Semanal & Diario \\
\cline { 2 - 6 } \multicolumn{1}{c}{ Sustancia } & $n(\%)$ & $n(\%)$ & $n(\%)$ & $n(\%)$ & $n(\%)$ \\
\hline Tabaco & $321(53,4)$ & $105(17,5)$ & $27(4,5)$ & $65(10,8)$ & $83(13,8)$ \\
Alcohol & $101(16,9)$ & $210(35,1)$ & $127(22,6)$ & $141(23,6)$ & $11(1,8)$ \\
Marihuana & $291(48,6)$ & $121(20,2)$ & $50(8,3)$ & $81(13,5)$ & $56(9,3)$ \\
Cocaína & $576(96,0)$ & $19(3,2)$ & $5(0,8)$ & -- & -- \\
Inhalantes & $591(98,5)$ & $4(0,7)$ & $4(0,7)$ & $1(0,2)$ & -- \\
Anfetaminas & $578(96,5)$ & $18(3,0)$ & $1(0,2)$ & $2(0,3)$ & -- \\
Tranquilizantes & $521(86,8)$ & $54(9,0)$ & $8(1,3)$ & $9(1,5)$ & $8(1,3)$ \\
Alucinógenos & $554(92,5)$ & $39(6,5)$ & $4(0,7)$ & $1(0,2)$ & $1(0,2)$ \\
Opiáceos & $575(96,0)$ & $18(3,0)$ & $5(0,8)$ & -- & $1(0,2)$ \\
Otras drogas & $550(91,5)$ & $15(2,5)$ & $4(0,7)$ & $6(1,0)$ & $9(1,5)$ \\
\hline
\end{tabular}

Nota. Otras drogas: relajantes, esteroides, anabólicos, adelgazantes, entre las más frecuentes. 
Tabla 6

Pruebas t de Student para Diferencias en el Consumo de Drogas por Tipo, según Sexo y Procedencia

\begin{tabular}{|c|c|c|c|c|c|c|c|}
\hline Droga & $M(D E)$ & $M(D E)$ & $t$ & $p$ & g.l. & $95 \%$ IC & $d$ de Cohen \\
\hline Sexo & Hombre & Mujer & & & & & \\
\hline Tabaco & $1,31(1,59)$ & $1,03(1,42)$ & 2,09 & 0,032 & 414 & {$[0,01,0,52]$} & 0,18 \\
\hline Alcohol & $1,78(1,15)$ & $1,47(1,02)$ & 3,38 & $<0,001$ & 410 & {$[0,13,0,50]$} & 0,28 \\
\hline Marihuana & $1,40(1,54)$ & $0,99(1,27)$ & 3,35 & $<0,001$ & 385 & {$[0,17,0,65]$} & 0,29 \\
\hline Cocaína & $0,06(0,26)$ & $0,04(0,24)$ & 1,13 & 0,259 & 423 & & \\
\hline Tranquilizantes & $0,15(0,56)$ & $0,26(0,72)$ & $-2,06$ & 0,040 & 547 & {$[-021,-0,00]$} & 0,17 \\
\hline Alucinógenos & $0,15(0,48)$ & $0,06(0,26)$ & 2,54 & 0,012 & 290 & {$[0,02,0,16]$} & 0,23 \\
\hline Procedencia & Urbano & Rural & & & & & \\
\hline Tabaco & $1,21(1.52)$ & $0,68(1,23)$ & 3,25 & 0,002 & 97 & {$[0,20,0,85]$} & 0,38 \\
\hline Alcohol & $1,63(1,07)$ & $1,23(1,09)$ & 2,90 & 0,004 & 593 & {$[012,0,66]$} & 0,37 \\
\hline Marihuana & $1,18(1,39)$ & $0,88(1,36)$ & 1,67 & 0,096 & 594 & & \\
\hline Cocaína & $0,05(0,26)$ & $0,03(0,17)$ & 0,69 & 0,491 & 595 & & \\
\hline Tranquilizantes & $0,23(0,69)$ & $0,12(0,44)$ & 1,86 & 0,065 & 118 & & \\
\hline Alucinógenos & $0,09(0,37)$ & $0,06(0,24)$ & 0,81 & 0,418 & 594 & & \\
\hline
\end{tabular}

Nota. El análisis se hizo con las drogas más consumidas.

\section{Análisis de Senderos}

Se estimó el puntaje total de las subescalas de Depresión, Ansiedad y Estrés, los cuales fueron los indicadores utilizados para el análisis de senderos, el cual propuso un efecto directo de Ansiedad en Estrés e indirecto a través de Depresión. En cuanto a los efectos directos, Ansiedad y Depresión se asociaron con Estrés. Además, Ansiedad se asoció con Depresión. También hubo un efecto indirecto, es decir, un efecto de Ansiedad en Estrés mediado por Depresión, $\beta=0,209, p=0,001$, dando un efecto total de $\beta=0,722, p=0,001$. El $r^{2}$ del modelo es 0,553 , es decir, explica un $55 \%$ de la varianza de la variable Estrés (ver Figura 1).

\section{Figura 1}

Modelo de Mediación con Efecto Directo e Indirecto de Ansiedad en Estrés, con Depresión como Mediador.

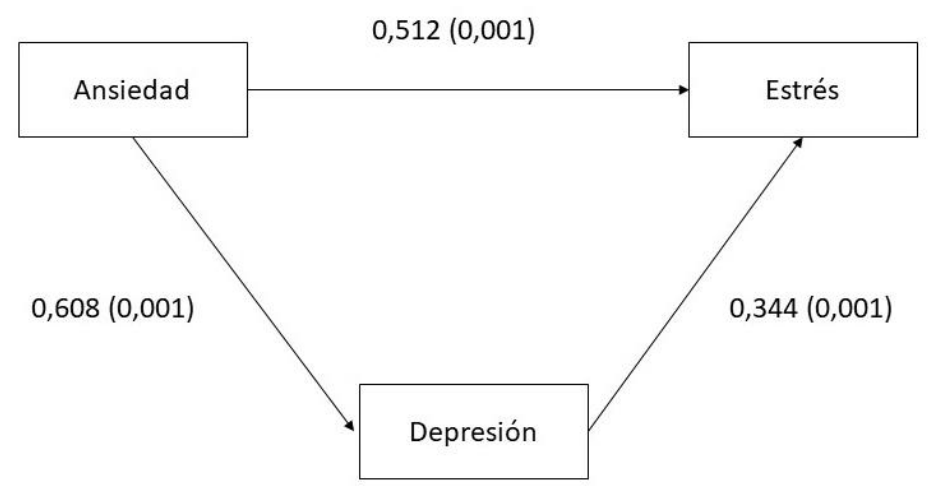

Nota. Los valores corresponden a los coeficientes Beta estandarizados (B) y los valores entre paréntesis corresponden al valor $p$. 


\section{Discusión}

Esta investigación permite una visión actualizada de la sintomatología y hábitos de salud mental en una muestra de universitarios chilenos y, aun cuando los resultados corresponden a un tamizaje de sintomatología en un periodo de tiempo limitado y no a un diagnóstico de cuadros clínicos, refleja un escenario preocupante, puesto que corrobora que los estudiantes que transitan por la adultez emergente presentarían una elevada prevalencia de psicopatología, incluso mayor a la reportada para la población general, teniendo, a su vez, comorbilidades de riesgo en salud mental (Arrieta Vergara et al., 2014; Baader et al., 2014; Micin \& Bagladi, 2011).

En específico, esta investigación deja en evidencia que más de un 40\% de los participantes de este estudio presenta sintomatología depresiva, ansiosa y/o de estrés y casi un tercio de los estudiantes presenta estos tres cuadros sintomatológicos de forma conjunta. Estos hallazgos reafirman la vulnerabilidad de los universitarios ante el desarrollo de trastornos emocionales (Antúnez \& Vinet, 2012) y son coherentes con la prevalencia a nivel nacional, que demuestra que la depresión y ansiedad representan los problemas de salud mental más frecuentes en la adultez emergente (Antúnez \& Vinet, 2013; Arrieta Vergara et al., 2014; Baader et al., 2014; Micin \& Bagladi, 2011). Por su parte, las altas tasas de estrés pueden comprenderse por la percepción que tienen muchos jóvenes de estar sobrepasados por las exigencias, a raíz de los múltiples estresores psicosociales que deben enfrentar para adaptarse a su nuevo contexto vital, así como también por las demandas propias del entorno universitario (Antúnez \& Vinet, 2013; Baader et al., 2014). Al respecto, hay evidencia que sostiene que estos estresores afectan especialmente a los jóvenes que tienen problemáticas previas de salud mental, lo cual acentúa la importancia de fortalecer los recursos y herramientas personales de los estudiantes, para promover su afrontamiento apropiado a los estresores de la vida y las presiones de los estudios universitarios (Cova Solar et al., 2007).

Asimismo, este estudio ofrece algunos resultados adicionales sobre la relación entre depresión, ansiedad y estrés, corroborando la existencia de efectos directos y un efecto indirecto de la Ansiedad en Estrés mediado por Depresión. Dicha información es coherente con lo expresado en la literatura respecto a las características comunes de estos cuadros y la comorbilidad con la que tienden a presentarse, lo cual aumenta la complejidad clínica de esta sintomatología, pudiendo implicar una disminución de la efectividad de tratamientos habituales y un peor pronóstico (Antúnez \& Vinet, 2012). Frente a esto, y teniendo en consideración que los análisis de mediación presentados en este estudio son una exploración preliminar del tema, se resalta la importancia de profundizar este modelo, considerando las implicancias para el desarrollo de intervenciones en salud mental y el manejo de tratamientos más efectivos y especializados.

De forma adicional, esta investigación apoya la idea de que las prevalencias en salud mental pueden verse influenciadas por la presencia de determinadas variables sociodemográficas. Al respecto, un hallazgo llamativo de este estudio es que las mujeres presentaron un porcentaje más alto de síntomas de ansiedad y estrés que los hombres, mientras que en depresión no se encontraron diferencias por sexo. Este resultado concuerda con la reciente investigación desarrollada por San Martín (2018), en cuya muestra de 1469 universitarios chilenos se observa la misma tendencia respecto de la experimentación de estos cuadros de ansiedad y estrés. Una forma de comprender estos resultados es que la ansiedad constituye uno de los componentes afectivos del proceso de estrés (Antúnez \& Vinet, 2012), por lo cual es esperable que la prevalencia de ambos síntomas sea similar. A su vez, la mayor prevalencia de estos síntomas en mujeres puede entenderse a partir de la hipótesis de la intensidad emocional, que consiste en que las mujeres tienden a reconocer, comunicar y experimentar en mayor medida sus estados emocionales, ya sea negativos o positivos, lo cual ha implicado que en la literatura ellas figuren con niveles más altos de malestar emocional, pero también con más emociones positivas, bienestar y satisfacción (Cova Solar, 2004).

Un resultado llamativo corresponde a la inexistencia de diferencias según sexo en la prevalencia de depresión, puesto que investigaciones previas desarrolladas con universitarios chilenos indican que son las mujeres quienes poseen niveles mayores de depresión (Antúnez \& Vinet, 2013; Baader et al., 2014; Cova Solar et al., 2007). Frente a ello, Dávila Figueras et al. (2011) señalan que la depresión se asocia a experiencias de vida más complejas que dependen de diversos factores genéticos y psicosociales, por lo cual es comprensible que hombres y mujeres puedan experimentar en similar medida estos síntomas; no obstante, es necesario seguir profundizando en este hallazgo.

En relación a la influencia de otras variables sociodemográficas, este estudio subraya que los jóvenes provenientes de zonas urbanas de esta muestra presentan mayores tasas de ansiedad, mientras que el NSE 
no impactaría en el desarrollo de sintomatología depresiva, ansiosa o de estrés, al menos en esta muestra. Esto último constituye un resultado relevante, considerando que investigaciones previas en el área indican que condiciones socioculturales, como la ruralidad o pertenecer a estratos socioeconómicos bajos, son considerados factores de riesgo para el desarrollo de alteraciones en salud mental (Antúnez \& Vinet, 2013; Baader et al., 2014). Frente a este escenario, es necesario tener en cuenta que el nuevo sistema de becas y créditos universitarios en Chile no solo ha promovido un incremento significativo de la matrícula en educación superior, sino también ha propiciado un cambio en el perfil del estudiante, facilitando un acceso más equitativo de jóvenes de distinta procedencia y NSE (Baader et al., 2014; Micin \& Bagladi, 2011). Considerando lo anterior, se podría hipotetizar que la universidad es un contexto que impone tareas y exigencias comunes en los estudiantes, lo cual puede uniformar las condiciones de la vida cotidiana bajo las cuales se desenvuelven, disminuyendo el impacto de las condiciones sociales de la familia de origen, tales como la procedencia y el NSE, en el desarrollo de sintomatología de salud mental.

Otro resultado que se torna especialmente alarmante es que el 5,1\% de los estudiantes de este estudio ha presentado IS en las últimas dos semanas. Estas cifras son similares a investigaciones previas con universitarios chilenos (Baader et al., 2014; Cova Solar et al., 2007; Micin \& Bagladi, 2011) y reafirman una realidad inquietante, puesto que el suicidio se ha convertido en la segunda causa de muerte en el rango etario de los 15 a 29 años (Organización Panamericana de la Salud, 2014). Por esta razón, resultan altamente relevantes los esfuerzos dirigidos a realizar un diagnóstico oportuno, más aún considerando que el suicidio es una de las principales causas de muerte prematura prevenibles (Baader et al., 2014; Micin \& Bagladi, 2011) y que la IS presenta una elevada comorbilidad con los trastornos del ánimo (Baader et al., 2014).

Otra arista estudiada en esta investigación corresponde a los hábitos de salud relacionados con la alimentación, patrones de sueño y consumo de sustancias. En relación a la alimentación, el escenario que revelan los datos es preocupante, ya que, al sumar la prevalencia obtenida en conductas alimentarias de riesgo moderado y alto, se observa que la mitad de los estudiantes presenta comportamientos que pueden derivar en un TCA. Cabe destacar que esta cifra es considerablemente más alta a las reportadas por otros estudios sobre la prevalencia de TCA en universitarios chilenos (Baader et al., 2014; Mediano-Stoltze et al., 2013; Micin \& Bagladi, 2011). No obstante, la menor prevalencia que reportan dichas investigaciones puede deberse a que los estudios citados han evaluado la presencia de cuadros clínicos, por lo que es posible que el porcentaje sea mayor cuando se trata de conductas de riesgo que aún no configuran un trastorno, tales como las que se midieron en esta investigación. Esto concuerda con lo señalado por Micin \& Bagladi (2011), quienes destacan que la incidencia de TCA es relativamente escasa en la población; sin embargo, esto aumenta cuando se considera a aquellas personas que presentan conductas alimentarias de riesgo o que no cumplen todos los criterios diagnósticos de un TCA, lo cual representa la fase previa para su desarrollo.

Asimismo, los resultados obtenidos en este estudio dan cuenta que las mujeres poseen mayores conductas alimentarias de riesgo a nivel general, lo cual concuerda con otros estudios chilenos (Baader et al., 2014; Mediano-Stoltze et al., 2013; Micin \& Bagladi, 2011). Frente a esto, cabe señalar que la evidencia disponible es consistente respecto a que las mujeres presentan mayores variables cognoscitivas y comportamentales de riesgo para el desarrollo de TCA, tales como mayores niveles de insatisfacción corporal, distorsión respecto a la percepción correcta de su cuerpo, preocupación por el peso y realización de conductas restrictivas para alcanzar ideales sociales de delgadez (Durán et al., 2013; Escolar-Llamazares et al., 2017; Mediano-Stoltze et al., 2013).

Por otro lado, este estudio refuerza el hecho de que la cantidad y calidad de sueño es uno de los mayores problemas de salud para los jóvenes, dada su prevalencia, curso clínico prolongado, tendencia a la cronificación y a presentarse en comorbilidad con otros problemas de salud (Durán-Agüero et al., 2016; Durán et al., 2017). Esto se evidencia en que el promedio de horas de sueño que reportan los participantes es menor a los parámetros saludables recomendados ( 7 a 8 horas) y que tanto hombres como mujeres presentan síntomas asociados al insomnio y la hipersomnia, lo cual concuerda con estudios previos que indican que la privación de sueño es un problema común en universitarios (Durán-Agüero et al., 2016). Un resultado llamativo consiste en que los estudiantes provenientes de zonas urbanas presentaron mayores puntajes de hipersomnia, lo cual sugiere que el entorno urbano podría ser un factor favorecedor de este tipo de problemas, al considerar variables que pueden contribuir a la privación de sueño y desencadenar hipersomnia, tales como contaminación acústica, uso de dispositivos electrónicos antes de dormir o pobre estructuración de horarios de sueño, hipótesis que requiere de mayor estudio. La información recabada se torna relevante, considerando que el sueño es un elemento fundamental para mantener un óptimo funcionamiento cognitivo y físico y, además, tiene una función importante en la autorregulación emocional, por lo que las dificultades en este 
ámbito se han asociado con mayores síntomas depresivos, ansiedad y consumo de sustancias (Durán-Agüero et al., 2016).

Finalmente, respecto al consumo de sustancias, si bien las cifras no son tan altas como las reportadas en estudios previos (Instituto Nacional de la Juventud [INJUV], 2015; SENDA, 2019), siguen siendo preocupantes, considerando la amplia variabilidad de drogas consumidas y su frecuencia. Un hallazgo importante fue que la marihuana se posicionó como la segunda droga más consumida de forma ocasional, mensual o semanal por parte de los universitarios. Hasta hace unos años, la tendencia indicaba que el tabaco era la droga más consumida después del alcohol (INJUV, 2015); no obstante, estudios más recientes muestran el incremento del consumo de marihuana, incluso por sobre el tabaco (San Martín, 2018). Lo anterior puede comprenderse porque drogas como esta última en la población joven son observadas como químicas y más dañinas (INJUV, 2015), sin embargo, la percepción de riesgo asociada al consumo de marihuana ha disminuido, considerándola entre los jóvenes como una droga natural, "más sana", poco o nada adictiva, consumida usualmente en espacios de socialización entre pares, lo cual podría favorecer un mayor consumo (Cazenave et al., 2017; INJUV, 2015).

Adicionalmente, este estudio confirma que los hombres presentan un consumo más alto de alcohol, tabaco, marihuana y alucinógenos que las mujeres. Lo anterior coincide con la evidencia nacional (INJUV, 2015) y con investigaciones que apuntan a una mayor aceptación social del consumo en hombres, además de una mayor experimentación y una menor percepción de riesgo (Banderas Rodríguez et al., 2010; Sánchez Pardo, 2012). Por su parte, las mujeres de esta muestra presentaron un mayor consumo de tranquilizantes, lo que coincide con investigaciones previas que lo indican en casi todos los países de América, especialmente en la etapa universitaria (Ahumada et al., 2019), siendo las que reconocen mayor sintomatología de salud mental, lo que conlleva a la búsqueda de ayuda profesional, accediendo, de este modo, al uso de tranquilizantes, como una forma de sobrellevar estas problemáticas (Droguett et al., 2019).

Asimismo, en esta muestra se corrobora que la residencia urbana y pertenecer a NSE más altos son factores que podrían relacionarse con un mayor consumo de alcohol. Lo anterior ha sido comprendido a partir de la mayor accesibilidad a drogas que puede conllevar el vivir en la ciudad, así como también porque la dinámica campo-ciudad que mantienen muchos jóvenes que viajan regularmente a la ciudad puede repercutir en un menor consumo, debido a la necesidad de cumplir con horarios de buses y, en muchos casos, contribuir al trabajo familiar (Alarcón et al., 2018). En relación al NSE, la evidencia refiere que los jóvenes de estratos socioeconómicos altos presentan un mayor consumo de alcohol (Betancourth-Zambrano et al., 2017; Landero Hernández \& Villarreal González, 2007), lo cual podría relacionarse a que este grupo cuenta con mayor poder adquisitivo que facilita la compra y el acceso al alcohol. Sin embargo, esta hipótesis requiere mayor estudio.

En cuanto a las limitaciones del estudio, es necesario tener en cuenta que, dadas las dificultades para poder acceder a todas las universidades a nivel nacional, se optó por la utilización de un muestreo no probabilístico, lo cual restringe la generalización de los resultados y los hace representativos solo de la muestra de estudio. De acuerdo a ello, como futuros lineamientos se propone realizar estudios de prevalencia de salud mental en universitarios a nivel nacional, que utilicen un muestreo probabilístico donde se aplique masivamente la $1^{\text {a }}$ Encuesta de Salud Mental Universitaria y que, a su vez, puedan incluir distintos tipos de instituciones (universidades públicas y privadas, centros de formación técnica e institutos profesionales).

Otra limitación es que esta investigación evaluó sintomatología de salud mental a través de cuestionarios de autorreporte, lo que podría explicar que las cifras recabadas sean un tanto más elevadas que los estudios que han medido prevalencia de cuadros clínicos de salud mental. Futuras investigaciones podrían incluir instrumentos de entrevista clínica tales como la Entrevista Diagnóstica Internacional Compuesta (CIDI, por sus siglas en inglés; División de Salud Mental, 1997), una entrevista altamente estructurada que entrega diagnósticos psiquiátricos tanto actuales como presentes a lo largo de la vida, de acuerdo a criterios del CIE-10 y al DSM-IV, para así tener uniformidad de métodos de evaluación en salud mental.

Adicionalmente, constituye una limitación el empleo de algunos instrumentos no validados en Chile, ya que el comportamiento de los ítems puede variar en función de la cultura, sugiriendo que las conclusiones de este estudio deban ser acogidas con cautela. Sin embargo, es importante considerar que los estudios de validación reportados para los instrumentos no validados en Chile han sido desarrollados con población latinoamericana o hispanohablante, contextos cercanos a la realidad chilena, lo cual podría explicar que una buena parte de los instrumentos en este estudio obtuvo adecuados indicadores de consistencia interna. Para el caso del CBCAR y el ASSIST, los niveles de consistencia interna estuvieron por debajo de lo esperado, por lo cual los resultados derivados de estas escalas deben ser tomados con cautela. No obstante, es importante 
destacar que en el caso del CBCAR, los menores niveles de confiabilidad se obtuvieron para dos de sus dimensiones y no para la escala total, que sí obtuvo una adecuada consistencia interna, lo cual apoya el uso de sus resultados, considerando que la escala fue tratada de forma global y no por dimensión.

Otra limitación corresponde a la distribución de la muestra según su procedencia, pues la mayor parte de ella provenía de entornos urbanos. Por tal razón, los análisis de comparación de grupos con esta variable deben ser interpretados como un primer análisis a confirmar. Futuros estudios podrían contar con una muestra más amplia de procedencia rural para confirmar los resultados obtenidos según esta variable sociodemográfica.

Frente a este inquietante escenario actual es que se hace especialmente relevante el rol de las instituciones de salud y las de educación superior en materia de salud mental de los estudiantes. Actualmente Chile cuenta con un plan nacional de salud mental, sin embargo, dada la alta prevalencia de distintas patologías en la población general (Departamento de Epidemiología, 2018), se hace necesario contar con una ley de salud mental que contemple la cobertura de distintas patologías y el acceso a servicios de salud mental de distintos grupos etarios, para garantizar un adecuado tratamiento y prevención en todos sus niveles, en pro del bienestar de sus ciudadanos en sus distintas etapas de vida.

Respecto a las universidades, estas se convierten en muchos casos en el único espacio donde los jóvenes cuentan con servicios de apoyo social, académico y médico (Álamo et al., 2017). Las universidades a nivel nacional e internacional se han visto en la necesidad de ofrecer servicios de atención psicológica en sus centros de salud universitarios, desde orientación, intervención en crisis, hasta psicoterapia. Lo anterior, sin duda, permite que los estudiantes reciban un apoyo psicológico durante su trayectoria académica, sin embargo, en la mayor parte de las instituciones la demanda de atenciones supera la oferta de servicios de atención psicológica. Es por esta razón que los resultados de este estudio refuerzan la necesidad de desarrollar intervenciones atingentes en materias de promoción, prevención y tratamiento en salud mental, propiciando una focalización en las patologías más frecuentes (ansiedad, depresión y estrés) y que permitan observar la interacción de los cuadros de salud mental con variables sociodemográficas como el sexo, el NSE o la procedencia de los estudiantes. Este tipo de intervenciones focalizadas y diferenciadas permitiría favorecer el desarrollo personal y profesional integral de los jóvenes desde el ingreso de los estudiantes al entorno universitario (Antúnez \& Vinet, 2013; Baader et al., 2014).

Finalmente, todos los hallazgos expuestos en este estudio enfatizan la importancia de profundizar en el estado de salud mental y los hábitos de salud de los universitarios, considerando que la mayoría de los trastornos psicológicos que afectan la vida adulta de los sujetos tienen su primera aparición en la adolescencia y/o en la adultez emergente (Álamo et al., 2017); esta última etapa es para muchos jóvenes el momento en que comienzan a asumir la responsabilidad sobre las prácticas que pueden reforzar o dañar su salud, por lo cual se convierte en una etapa crucial para establecer comportamientos y hábitos saludables para etapas futuras de su desarrollo vital (Arrieta Vergara et al., 2014; Durán et al., 2017).

\section{Referencias}

Adimark (2000). El nivel socio económico Esomar: manual de aplicación. https://vdocuments.mx/el-nivel-socio-economico-esomarmanual-de-aplicacion-adimark-santiago-octubre.html

Ahumada, G., Araneda, J. C., Clarke, P., Cumsille, F. \& Hynes, M. (2019). Informe sobre el consumo de drogas en las Américas 2019. Organización de los Estados Americanos, Secretaría de Seguridad Multidimensional, Comisión Interamericana para el Control del Abuso de Drogas. http://www.cicad.oas.org/main/pubs/Informe\%20sobre\%20el\%20consumo\%20de\%20drogas\%20en\%20las\%20Am\%C3\%A9ricas\%202019.pdf

Álamo, C., Bejer, T., Bagladi, V., Cárcamo, N., San Martín, D. \& Fischer, M. (2017). Perfil integrativo de pacientes de salud mental atendidos en el Centro de Salud Universitario de la Universidad Austral de Chile. Actualizaciones en Psicoterapia Integrativa, 9, 9-20. https://icpsi.cl/wp-content/uploads/2018/03/AcPI_2017.pdf

Alarcón, A. M., Muñoz, S. \& Grandjean, M. (2018). Consumo de alcohol en escolares de un territorio de la Araucanía-Chile: etnicidad y residencia. Revista Chilena de Pediatría, 89(4), 454-461. https://doi.org/10.4067/S0370-41062018005000601

Altamirano Martinez, M. B., Vizmanos Lamotte, B. \& Unikel Santoncini, C. (2011). Continuo de conductas alimentarias de riesgo en adolescentes de México. Revista Panamericana de Salud Pública, 30(5), 401-407. https://doi.org/10.1590/S1020-49892011001100001

Antúnez, Z. \& Vinet. E. V. (2012). Escalas de depresión, ansiedad y estrés (DASS - 21): validación de la versión abreviada en estudiantes universitarios chilenos. Terapia Psicológica, 30(3), 49-55. https://doi.org/10.4067/S0718-48082012000300005

Antúnez, Z. \& Vinet, E. V. (2013). Problemas de salud mental en estudiantes de una universidad regional chilena. Revista Médica de Chile, 141(2), 209-216. https://doi.org/10.4067/S0034-98872013000200010

Área de Desarrollo Sostenible y Salud Ambiental. (2011). La prueba de detección de consumo de alcohol, tabaco y sustancias (ASSIST): manual para uso en la atención primaria. Organización Mundial de la Salud, Organización Panamericana de la Salud. https://apps.who.int/iris/handle/10665/85403

Arnett, J. J. (2000). Emerging adulthood: A theory of development from the late teens through the twenties. American Psychologist, 55(5), 469-480. https://doi.org/10.1037/0003-066X.55.5.469 
Arnett, J. J. (2004). Emerging adulthood: The winding road from late teens through twenties. Oxford University Press. https://doi.org/10.1093/acprof:oso/9780199929382.001.0001

Arnett, J. J. (2007/2008). Adolescencia y adultez emergente: un enfoque cultural (3a ed.; M. E. Ortiz, Trad.). Pearson Educación.

Arnett, J. J., Žukauskienè, R. \& Sugimura, K. (2014). The new life stage of emerging adulthood at ages 18-29 years: Implications for mental health. The Lancet Psychiatry, 1(7), 569-576. https://doi.org/10.1016/S2215-0366(14)00080-7

Arrieta Vergara, K. M., Díaz Cárdenas, S. \& González Martínez, F. (2014). Síntomas de depresión y ansiedad en jóvenes universitarios: prevalencia y factores relacionados. Revista Clínica de Medicina de Familia, 7(1), 14-22. https://doi.org/10.4321/S1699-695X2014000100003

Auerbach, R. P., Alonso, J., Axinn, W. G., Cuijpers, P., Ebert, D. D., Green, J. G., Hwang, I., Kessler, R.C., Liu, H., Mortier, P., Nock, M. K., Pinder-Amaker, S., Sampson, N. A., Aguilar-Gaxiola, S., Al-Hamzawi, A., Andrade, L. H., Benjet, C., Caldas-de-Almeida, J. M., Demyttenaere, K. ... Bruffaerts, R. (2016). Mental disorders among college students in the World Health Organization world mental health surveys. Psychological Medicine, 46(14), 2955-2970. https://doi.org/10.1017/S0033291716001665

Baader, T., Rojas, C., Molina, J. L., Gotelli, M., Alamo, C., Fierro, C., Venezian, S. \& Dittus, P. (2014). Diagnóstico de la prevalencia de trastornos de la salud mental en estudiantes universitarios y los factores de riesgo emocionales asociados. Revista Chilena de NeuroPsiquiatría, 52(3), 167-176. https://doi.org/10.4067/S0717-92272014000300004

Banderas Rodríguez, C. R., Martínez Chacón, A. \& Romo González, T. (2010). Prevención integral de consumo de alcohol y drogas en estudiantes universitarios: una propuesta de intervención grupal. Acta Colombiana de Psicología, 13(2), 19-33. https://actacolombianapsicologia.ucatolica.edu.co/article/view/367/372

Barraza, R., Muñoz, N., Alfaro, M., Álvarez, Á., Araya, V., Villagra, J. \& Contreras, A. M. (2015). Ansiedad, depresión, estrés y organización de la personalidad en estudiantes novatos de medicina y enfermería. Revista Chilena de Neuro-Psiquiatría, 53(4), 251260. https://doi.org/10.4067/S0717-92272015000400005

Barrera-Herrera, A. \& Vinet, E. V. (2017). Adultez emergente y características culturales de la etapa en universitarios chilenos. Terapia Psicológica, 35(1), 47-56. https://doi.org/10.4067/S0718-48082017000100005

Betancourth-Zambrano, S., Tacán-Bastidas, L. \& Córdoba-Paz, E. G. (2017). Consumo de alcohol en estudiantes universitarios colombianos. Universidad y Salud, 19(1), 37-50. https://doi.org/10.22267/rus.171901.67

Bobes García, J., González G-Portilla, M. P., Sáiz Martínez, P. A., Bascarán Fernández, M. T., Iglesias Alvarez, C. \& Fernández Domínguez, J. M. (2000). Propiedades psicométricas del Cuestionario Oviedo de Sueño. Psicothema, 12(1), $107-112$. http://www.psicothema.com/psicothema.asp?id=260

Cazenave, A., Saavedra, W., Huerta, P., Mendoza, C. \& Aguirre, C. (2017). Consumo de marihuana en jóvenes universitarios: percepción de los pares. Ciencia y Enfermería, 23(1), 15-24. https://doi.org/10.4067/S0717-95532017000100015

Cova Solar, F. (2004). Diferencias de género en bienestar y malestar emocional: evidencias contradictorias. Terapia Psicológica, 22(2), 165-169. https://www.redalyc.org/articulo.oa?id=78522207

Cova Solar, F., Alvial, W., Aro, M., Bonifetti, A., Hernández, M. \& Rodríguez, C. (2007). Problemas de salud mental en estudiantes de la Universidad de Concepción. Terapia Psicológica, 25(2), 105-112. https://doi.org/10.4067/S0718-48082007000200001

Dávila Figueras, A., Ruiz Celis, R., Moncada Arroyo, L. \& Gallardo Rayo, I. (2011). Niveles de ansiedad, depresión y percepción de apoyo social en estudiantes de Odontología de la Universidad de Chile. Revista de Psicología de la Universidad de Chile, 20(2), 147-172. https://doi.org/10.5354/0719-0581.2011.17937

Departamento de Epidemiología. (2018, Enero). Encuesta nacional de salud 2016-2017: segunda entrega de resultados. Gobierno de Chile, Ministerio de Salud, Subsecretaría de Salud Pública, División de Planificación Sanitaria. http://www.minsal.cl/wpcontent/uploads/2018/01/2-Resultados-ENS_MINSAL_31_01_2018.pdf

Departamento de Salud Mental y Abuso de Sustancias. (2004). Invertir en salud mental. Organización Mundial de la Salud. https://www.who.int/mental_health/advocacy/en/spanish_final.pdf

División de Salud Mental. (1997). Entrevista Diagnostica Internacional Compuesta (CIDI): (versión medular 2.1), versión de 12 meses. Organización Mundial de la Salud. https://docplayer.es/21133851-Entrevista-diagnostica-internacional-compuesta-cidi.html

Droguett, N., Vidal, C., Medina, B. \& Hoffmeister, L. (2019). Factores asociados al consumo de psicofármacos sin receta en Chile: estudio descriptivo basado en la Encuesta Nacional de Consumo de Drogas en Población General. Medwave, 19(6), Artículo 7670. https://doi.org/10.5867/medwave.2019.06.7670

Durán, S., Crovetto, M., Espinoza, V., Mena, F., Oñate, G., Fernández, M., Coñuecar, S., Guerra, A. \& Valladares, M. (2017). Caracterización del estado nutricional, hábitos alimentarios y estilos de vida de estudiantes universitarios chilenos: estudio multicéntrico. Revista Médica de Chile, 145(11), 1403-1411. https://doi.org/10.4067/s0034-98872017001101403

Durán, S., Rodríguez, M. P., Record, J., Barra, R., Olivares, R., Tapia, A., Moraga, A., Campos, C., Mattar, P., Pérez, G. \& Neira, A. M. (2013). Autopercepción de la imagen corporal en estudiantes universitarios de Chile y Panamá. Revista Chilena de Nutrición, 40(1), 26-32. https://doi.org/10.4067/s0717-75182013000100004

Durán-Agüero, S., Fernández-Godoy, E., Fehrmann-Rosas, P., Delgado-Sánchez, C., Quintana-Muñoz, C., Yunge-Hidalgo, W., Hidalgo-Fernández, A. \& Fuentes-Fuentes, J. (2016). Menos horas de sueño asociado con mayor peso corporal en estudiantes de nutrición de una universidad chilena. Revista Peruana de Medicina Experimental y Salud Pública, 33(2), 264-268. https://doi.org/10.17843/rpmesp.2016.332.2100

Escolar-Llamazares, M. C., Martínez Martín, M. Á., González Alonso, M. Y., Medina Gómez, M. B., Mercado Val, E. \& Lara Ortega, F. (2017). Factores de riesgo de trastornos de la conducta alimentaria entre universitarios: estimación de vulnerabilidad por sexo y edad. Revista Mexicana de Trastornos Alimentarios, 8(2), 105-112. https://doi.org/10.1016/j.rmta.2017.05.003

Florenzano, R. (2005). Salud mental y características de personalidad de los estudiantes universitarios en Chile. Calidad en la Educación, 23, 105-114. https://doi.org/10.31619/caledu.n23.290

García-Portilla, M. P., Sáiz, P. A., Díaz-Mesa, E. M., Fonseca, E., Arrojo, M., Sierra, P., Sarramea, F., Sánchez, E., Goikole, J. M., Balanzá, V., Benabarre, A. \& Bobes, J. (2009). Rendimiento psicométrico del Cuestionario Oviedo de Sueño en pacientes con trastorno mental grave. Revista de Psiquiatría y Salud Mental, 2(4), 169-177. https://doi.org/10.1016/S1888-9891(09)73235-5

Instituto Nacional de la Juventud. (2017). 8va Encuesta nacional de juventud 2015. Gobierno de Chile, Ministerio de Desarrollo Social. http://biblioteca.digital.gob.cl/handle/123456789/1388

Jerez-Mendoza, M. \& Oyarzo-Barría, C. (2015). Estrés académico en estudiantes del Departamento de Salud de la Universidad de Los Lagos Osorno. Revista Chilena de Neuro-Psiquiatría, 53(3), 149-157. https://doi.org/10.4067/S0717-92272015000300002

Landero Hernández, R. \& Villarreal González, M. E. (2007). Consumo de alcohol en estudiantes en relación con el consumo familiar y de los amigos. Psicología y Salud, 17(1), 17-23. https://psicologiaysalud.uv.mx/index.php/psicysalud/article/view/731/1289 
Mediano-Stoltze, F., Repetto, P. B. \& Molina, Y. (2013). Insatisfacción corporal, creencias sobre control del peso y consumo de cigarrillos en jóvenes. Terapia Psicológica, 31(2), 155-163. https://doi.org/10.4067/S0718-48082013000200002

Mellor, D., Vinet, E. V., Xu, X., Bt Mamat, N. H., Richardson, B. \& Román, F. (2015). Factorial invariance of the DASS-21 among adolescents in four countries. European Journal of Psychological Assessment, 31(2), 138-142. https://doi.org/10.1027/1015-5759/a000218

Micin, S. \& Bagladi, V. (2011). Salud mental en estudiantes universitarios: incidencia de psicopatología y antecedentes de conducta suicida en población que acude a un servicio de salud estudiantil. Terapia Psicológica, 29(1), 53-64. https://doi.org/10.4067/\$0718-48082011000100006

Organización Mundial de la Salud. (2013). Plan de acción sobre salud mental 2013-2020.

Organización Panamericana de la Salud. (2014). Prevención del suicidio, un imperativo global: resumen ejecutivo. Organización Mundial de la Salud. https://www.who.int/mental_health/suicide-prevention/exe_summary_spanish.pdf?ua=1

Osman, A., Gutierrez, P. M., Jiandani, J., Kopper, B. A., Barrios, F. X., Linden, S. C. \& Truelove, R. S. (2003). A preliminary validation of the Positive and Negative Suicide Ideation (PANSI) Inventory with normal adolescent samples. Journal of Clinical Psychology, 59(4), 493-512. https://doi.org/10.1002/jclp.10154

Pérez-Villalobos, C., Bonnefoy-Dibarrat, C., Cabrera-Flores, A., Peine-Grandón, S., Macaya-Abarca, K., Baqueano-Rodríguez, M. \& Jiménez-Espinoza, J. (2012). Problemas de salud mental en alumnos universitarios de primer año de Concepción, Chile. Anales de Psicología, 28(3), 797-804. https://doi.org/10.6018/analesps.28.3.156071

Román, F., Santibáñez, P. \& Vinet, E. V. (2016). Uso de las Escalas de Depresión Ansiedad Estrés (DASS-21) como instrumento de tamizaje en jóvenes con problemas clínicos. Acta de Investigación Psicológica, 6(1), 2325-2336. https://doi.org/10.1016/S2007-4719(16)30053-9

Román Mella, F., Vinet, E. V. \& Alarcón Muñoz, A. M. (2014). Escalas de Depresión, Ansiedad y Estrés (DASS-21): adaptación y propiedades psicométricas en estudiantes secundarios de Temuco. Revista Argentina de Clínica Psicológica, 23(2), 179-190. https://www.redalyc.org/articulo.oa?id=281943265009

Rossi, J. L., Jiménez, J. P., Barros, P., Assar, R., Jaramillo, K., Herrera, L., Quevedo, Y., Botto, A., Leighton, C. \& Martínez, F. (2019). Sintomatología depresiva y bienestar psicológico en estudiantes universitarios chilenos. Revista Médica de Chile, 147(5), 579-588. https://doi.org/10.4067/S0034-98872019000500579

San Martín, Y. (2018). Rol mediador de los síntomas emocionales negativos en la relación entre las dimensiones de la adultez emergente y el consumo de drogas en hombres y mujeres (Tesis de magíster no publicada). Universidad de La Frontera.

Sánchez Pardo, L. (2012). Género y drogas: guía informativa. Diputación de Alicante, Área de Igualdad y Juventud. https://pnsd.sanidad.gob.es/profesionales/publicaciones/catalogo/bibliotecaDigital/publicaciones/pdf/GuiaGenero_Drogas.pdf

Schulenberg, J. E., Sameroff, A. J. \& Cicchetti, D. (2004). The transition to adulthood as a critical juncture in the course of psychopathology and mental health. Development and Psychopathology, 16(4), 799-806. https://doi.org/10.1017/S0954579404040015

Servicio Nacional para la Prevención y Rehabilitación del Consumo de Drogas y Alcohol. (2019). Primer estudio de drogas en educación superior. Principales resultados. Gobierno de Chile, Ministerio del Interior y Seguridad Pública. http://www.senda.gob.cl/wpcontent/uploads/2019/05/2019_05_23_PPT_Ed_SuperiorEstudiosOK.pdf

Soto-Brandt, G., Portilla Huidobro, R., Huepe Artigas, D., Rivera-Rei, Á., Escobar, M. J., Salas Guzmán, N., Canales-Johnson, A., Ibáñez, A., Martínez Guzmán, C. \& Castillo-Carniglia, Á. (2014). Evidencia de validez en Chile del Alcohol, Smoking and Substance Involvement Screening Test (ASSIST). Adicciones, 26(4), 291-302. https://doi.org/10.20882/adicciones.27

Stallman, H. M. \& Shochet, I. (2009). Prevalence of mental health problems in Australian university health services. Australian Psychologist, 44(2), 122-127. https://doi.org/10.1080/00050060902733727

Storrie, K., Ahern, K. \& Tuckett, A. (2010). A systematic review: Students with mental health problems-A growing problem. International Journal of Nursing Practice, 16(1), 1-6. https://doi.org/10.1111/j.1440-172x.2009.01813.x

Unikel-Santoncini, C., Bojórquez-Chapela, I. \& Carreño-García, S. (2004). Validación de un cuestionario breve para medir conductas alimentarias de riesgo. Salud Pública de México, 46(6), 509-515. https://doi.org/10.1590/S0036-36342004000600005

Villalobos-Galvis, F. H. (2010). Validez y fiabilidad del Inventario de Ideación Suicida Positiva y Negativa-PANSI, en estudiantes colombianos. Universitas Psychologica, 9(2), 509-520. https://doi.org/10.11144/Javeriana.upsy9-2.vfii

Fecha de recepción: Agosto de 2019.

Fecha de aceptación: Junio de 2020. 